\title{
Correction to: Pre-therapy Somatostatin Receptor-Based Heterogeneity Predicts Overall Survival in Pancreatic Neuroendocrine Tumor Patients Undergoing Peptide Receptor Radionuclide Therapy
}

Rudolf A. Werner, ${ }^{1,2}$ Harun Ilhan, ${ }^{3}$ Sebastian Lehner, ${ }^{3,4}$ László Papp, ${ }^{5}$ Norbert Zsótér, ${ }^{6}$ Imke Schatka, ${ }^{7}$ Dirk O. Muegge, ${ }^{2}$ Mehrbod S. Javadi, ${ }^{1}$ Takahiro Higuchi, ${ }^{2,8}$ Andreas K. Buck, ${ }^{2}$ Peter Bartenstein, ${ }^{3}$ Frank Bengel, ${ }^{9}$ Markus Essler, ${ }^{10}$ Constantin Lapa, ${ }^{2}$ Ralph A. Bundschuh ${ }^{10}$

\footnotetext{
${ }^{1}$ The Russell H. Morgan Department of Radiology and Radiological Science, Division of Nuclear Medicine and Molecular Imaging, Johns Hopkins University School of Medicine, Baltimore, MD, USA

${ }^{2}$ Department of Nuclear Medicine, University Hospital Würzburg, Würzburg, Germany

${ }^{3}$ Department of Nuclear Medicine, University Hospital, LMU Munich, Munich, Germany

${ }^{4}$ Ambulatory Healthcare Center Dr. Neumaier \& Colleagues, Radiology, Nuclear Medicine, Radiation Therapy, Regensburg, Germany

${ }^{5}$ Department of Nuclear Medicine, Medical University of Vienna, Vienna, Austria

${ }^{6}$ Mediso Medical Imaging Systems Ltd., Budapest, Hungary

${ }^{7}$ Department of Nuclear Medicine, Charité - Universitätsmedizin Berlin, Berlin, Germany

${ }^{8}$ Department of Bio Medical Imaging, National Cardiovascular and Cerebral Research Center, Suita, Japan

${ }^{9}$ Department of Nuclear Medicine, Hannover Medical School, Hannover, Germany

${ }^{10}$ Department of Nuclear Medicine, University Hospital Bonn, Sigmund-Freud-Str. 25, 53127, Bonn, Germany
}

Correction to: Mol Imaging Biol

https://doi.org/10.1007/s11307-018-1252-5

The article "Pre-therapy Somatostatin Receptor-Based Heterogeneity Predicts Overall Survival in Pancreatic Neuroendocrine Tumor Patients Undergoing Peptide Receptor Radionuclide Therapy," written by Rudolf A. Werner, Harun Ilhan, Sebastian Lehner, László Papp, Norbert Zsótér, Imke Schatka, Dirk O. Muegge, Mehrbod S. Javadi, Takahiro Higuchi, Andreas K. Buck, Peter Bartenstein, Frank Bengel, Markus Essler, Constantin Lapa, and Ralph A. Bundschuh was originally published electronically on the publisher's internet portal (currently SpringerLink) on July 16, 2018 without open access.

With the author(s)' decision to opt for Open Choice the copyright of the article changed on July 25, 2018 to (C) The Author(s) 2018 and the article is forthwith distributed under the terms of the Creative Commons Attribution 4.0 International License (http://creativecommons.org/licenses/by/4.0/), which permits use, duplication, adaptation, distribution and reproduction in any medium or format, as long as you give appropriate credit to the original author(s) and the source, provide a link to the Creative Commons license and indicate if changes were made. 